\title{
Role of MRI in assessment of perianal fistula
}

\section{Abstract}

Background and objective: Perianal fistulae commonly occur in middle-aged men. It is important to determine the presence of fistula tracks, its grading and presence of abscess or inflammation. This study aimed to assess the role of MRI in diagnosis and classification of perianal fistula.

Methods: This study included 20 patients with perianal sepsis that were referred to the MRI section of Radiology Department in Rizgary Teaching Hospital between August 2015 and April 2016. All patients underwent MRI examination.

Results: The mean \pm SD age of the 20 patients was $38.5 \pm 10.4$ years (range 22-55 years), of which 16 patients (80\%) were males and four patients (20\%) were females. Eighteen patients had a single external opening and two patients had two external opening. All the simple non-branching fistulas were successfully identified by axial and coronal planes of contrast enhanced T1Weighted image fast spin echo, of which five cases had simple intersphincter (G1) fistula and two had simple transphincter (G3), seven cases had intersphincter fistula either secondary tract or horse shoe and abscess (G2), five cases had complex transsphincter (G4). Two cases of complex G4 type had inflammation and edema in ischiorectal and ischioanal fossa and the internal openings were clearer in STIR T2axia.

Conclusion: MRI non-invasively offers important information about perianal fistula that can reduce surgical complications and postoperative recurrence of the disease. Every patient with suspicious perianal sepsis should undergo MRI examination to prevent complications and morbidity.

Keywords: MRI; Perianal fistula; Intersphincter; Transphincter

\section{Introduction}

A fistula is defined as a pathologic tract connecting two hollow organs, or one hollow organ and the skin. Sinuses are defined when only one hollow organ or skin in involved. ${ }^{1}$ 'Fistula' is the Latin word for a reed, pipe, or flute. ${ }^{2}$ The fistula is thought to be a result of anal gland obstruction several inflammatory conditions and events, including Crohn's disease, pelvic infection, tuberculosis, diverticulitis, trauma during childbirth, pelvic malignancy, and radiation therapy. ${ }^{2}$ Perianal fistulae occur in approximately 10 out of 10,000 persons. ${ }^{3}$ Perianal fistulae commonly occur in middle-aged men. ${ }^{4}$ The male-to-female ratio of $1.8: 1^{5}$ The anal canal is a cylindrical structure surrounded by two muscular layers, the internal and external sphincters. The internal sphincter is composed of smooth muscle, the fibers of which are continuous with the circular smooth muscle of the rectum. ${ }^{6}$ The external sphincter consists of striated muscle and has posterior attachments to the anococcygeal ligament and anterior attachments to the perineal body and urogenital diaphragm. It merges proximally with the puborectalis muscle, which then merges with the levator plate of the pelvic floor. ${ }^{7}$ The two sphincters are separated by the intersphincteric space, which contains fat, areolar tissue, and the longitudinal muscle. This space forms a natural plane

* Department of Surgery, College of Medicine, Hawler Medical University, Erbil, I raq. 
of lower resistance in which fistulas and pus can readily spread. ${ }^{8}$ Knowledge of the anatomy of the anal sphincter complex and surrounding spaces is crucial for image interpretation. ${ }^{9}$ The internal sphincter (a smooth muscle) is hypointense on T1W and T2W TSE images and is relatively hyperintense on fat-suppressed T2W images. It shows enhancement on postgadolinium T1W images. ${ }^{10}$ The external anal sphincter (a striated muscle) is clearly visualized on MRI. It is hypo intense on T1W, T2W, and fat-suppressed T2W images, and is bordered laterally by the fat in the ischioanal fossa. The coronal images depict the levator ani muscle, the identification of which is important to distinguish supra levator from infra levator infections. ${ }^{9}$ Surgeons describe the site and direction of fistulous tracks by referring to the "anal clock" that is, the view of the anal region with the patient in the lithotomy position usually used for fistula surgery. At 12 o'clock is the anterior perineum and at 6 o'clock, the natal cleft; 3 o'clock refers to the left lateral aspect, and 9 o'clock, to the right of the anal canal. Fortunately, these descriptions correspond exactly with the view of the anal canal on axial MR images, and it is helpful for surgical colleagues if the radiologists relate the MR imaging findings to the anal clock. ${ }^{11}$ Initial classification of perianal fistulas was based on surgical anatomy described by Parks et al. Depending on the location and course of the primary tract, perianal fistulae have been classified into the four types of inter-sphincteric, trans-sphincteric, supra -sphincteric and extra-sphincteric. ${ }^{12}$ The inter-sphincteric type is the commonest type (60-70\%) where the infection starts from an anal gland and develops in the inter-sphincteric plane, lying between the internal and external sphincters, without penetrating the external sphincter. It eventually ruptures onto the skin, thereby creating the fistula. Trans-sphincteric type (20-30\%) occurs when the intersphincteric infection penetrates the external sphincter to reach the ischioanal fossa and, eventually, the perianal skin. ${ }^{11}$ The St. James's University Hospital classification for MRI is an MRI-based grading system for perianal fistulas that was validated by surgically proved cases. ${ }^{12}$ The St James's University Hospital classification relates to the anatomy seen at MR images in both axial and coronal plane. This grading system deals not only with the primary tract but also with secondary tracts (branches) and associated abscesses, which is needed in pre-operative medical imaging. St. James' University Hospital Classification of perianal fistula is divided into five grades. Grade 1 includes simple linear intersphincteric fistula, while Grade 2 includes intersphincteric fistula with intersphincteric abscess or secondary fistulous tract confined by the external sphincter. Grade 3 includes transphincteric fistula penetrates both the internal and external anal sphincters, and extends through the ischiorectal or ischioanal fossae in its pathway to its cutaneous opening, Grade 4 includes transsphincteric fistula with abscess or secondary tract within the ischioanal or ischiorectal fossa, and Grade 5 includes supra levator and extrasphincteric fistula and encompass a variety of complex tracts. ${ }^{11,12}$ The MRI is performed for perianal fistulas to determine the relationship of any fistulous track to the sphincter complex. It helps in determining the involvement of the sphincter, whether the track traverses both layers of the sphincter (trans-sphincteric) or only the internal sphincter (intersphincteric) and identifying any secondary fistulous tracks and the sites of any abscess cavities so giving grading to the fistula. ${ }^{1,2}$ This study aimed to assess the role of MRI in diagnosis and classification of perianal fistula.

\section{Methods}

The present study included 20 patients with perianal sepsis. These patients were referred to the MRI section of Radiology Department in Rizgary Teaching Hospital from Surgery Outpatient Clinic in the same 
hospital during the period between August 2015 and April 2016 as they had clinical manifestations of suspected perianal sepsis, perianal discharge and with visible one or two external opening. All patients underwent MRI examinations on Siemens 1.5 Tesla MRI, Siemens Medical Systems, Avanto, Germany. Initial three plane images were obtained. On the sagittal images, the axial cuts of the T1\&T2weighted fast spin echo with fat suppression images were planed parallel to the pelvic diaphragm. Then on the same sagittal plane, the coronal cuts were planed parallel to the anal canal tilted forward from the vertical by approximately $45^{\circ}$, T1-weighted fast spin echo with fat suppression images and T2-weighted fast spin echo with fat suppression images. After obtaining the axial and coronal images, contrast material (Gd DPTgad) was injected IV with a dose of $0.1 \mathrm{mmol} / \mathrm{kg}$. Then the axial and coronal T1-weighted fast spin echo with fat suppression images was obtained. Statistical analysis was conducted by using the statistical package for the social sciences (version 18). Descriptive analysis was applied to calculate means, numbers and percentages.

\section{Results}

The mean \pm SD age of the 20 patients studied was $38.5 \pm 10.4$ years (range 22-55 years), of which 16 patients (80\%) were males and four patients (20\%) were females. Eighteen (90\%) patients had a single external opening, and two (10\%) patients had two external openings. All the cases were evaluated for the site of the primary tract and its ramifications, the presence/absence of external sphincter involvement, and the location of the internal openings and presence/absence of secondary abscess. In this study, all simple nonbranching fistulas were successfully identified by axial and coronal planes of contrast enhanced FS T1WFSE images. Five $(2.5 \%)$ cases had a simple intersphincter fistula and the internal openings were clearer in STIR T2axia coronal, and the fistula was more conspicuous in post contrast $\mathrm{T} 1$ fat suppression (Figure 1).

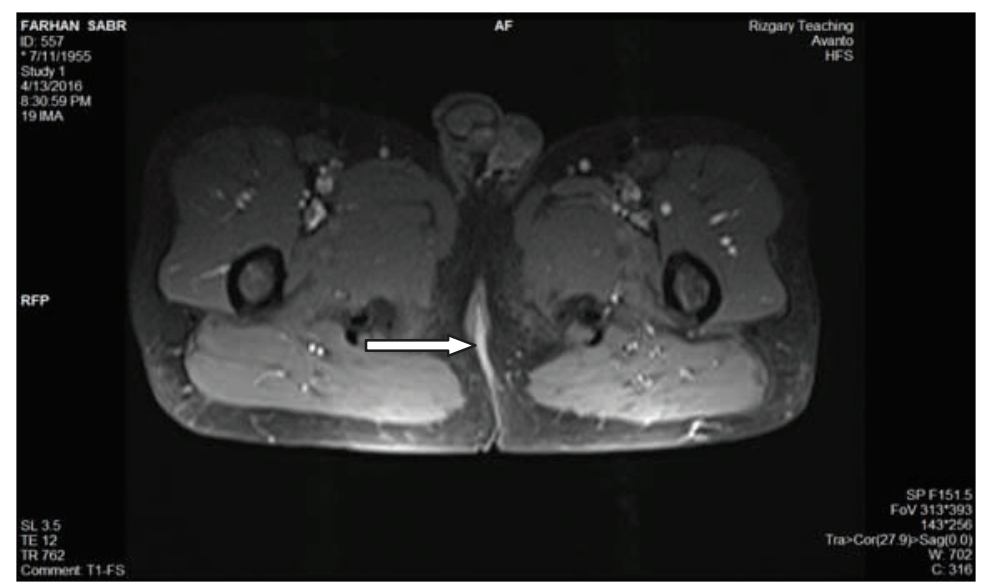

Figure 1: T1 axial fat suppression post contrast shows the intersphincteric fistula tract. 
Seven (35\%) cases had intersphentric fistula either secondary tract or horse shoe and abscess (G2) as shown in Figures 2 and 3 . Two (10\%) cases were simple transphincter fistula (G3) as shown in Figure 4. Five (25\%) cases had complex transsphincter fistula G4 (Figure 5).

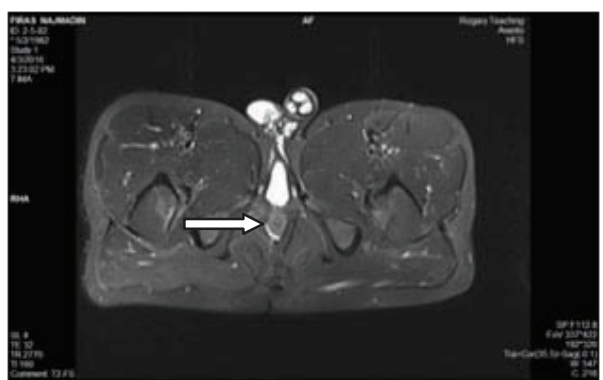

Figure 2: Axial T2 fat suppression-weighted image shows an intersphincteric horseshoe fistula Grade 2 (arrow).
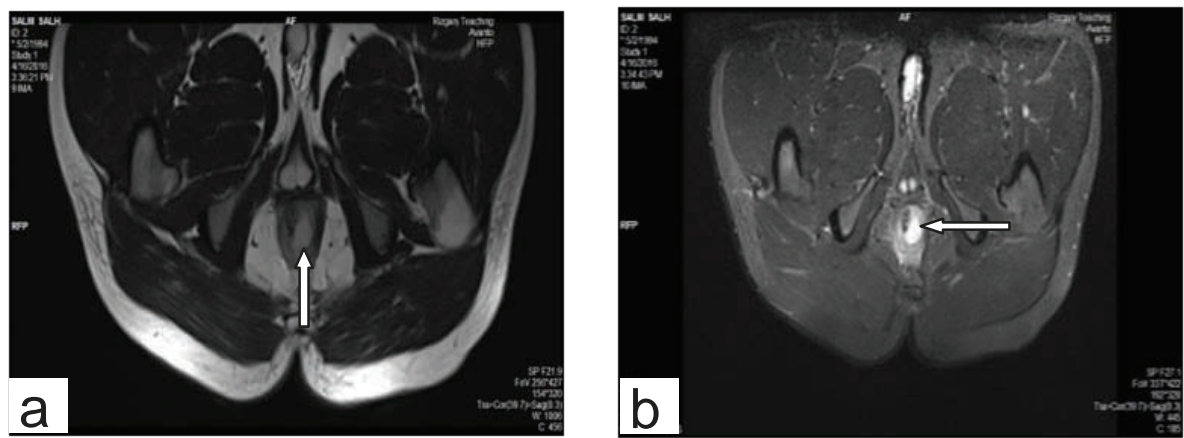

Figure 3: (a) Axial T1weighted image - and (b) T2 axial fat suppression shows an intersphincteric abscess.

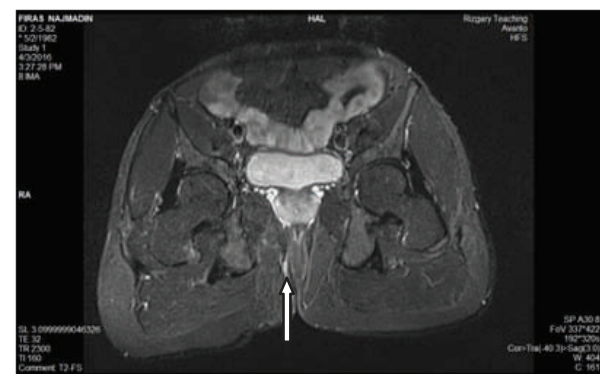

Figure 4: Coronal T2 fat suppression weighted images showing transsphincter fistula,
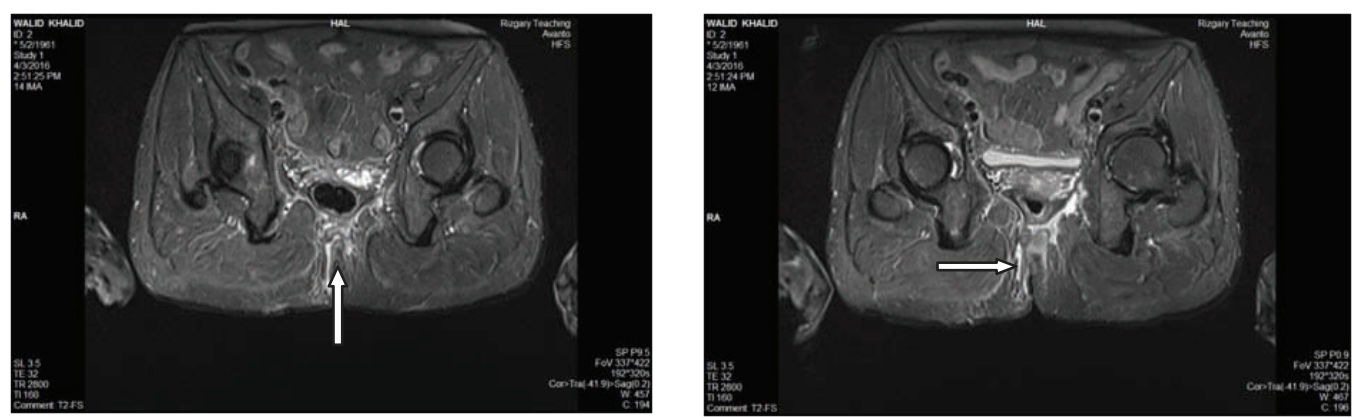

Figure 5: Axial T2 fat suppression with transsphincter with secondary tract. 
No cases of G5 were reported. One case was perianal sinus. Two cases of complex type G4 had inflammation and edema in ischiorectal and ischioanal fossa (Figure 6). The course and thickness of fistula tract were more clear in coroanal fat suppression rather than axial fat suppression (Figure 7). Regarding the distribution of MRI perianal fistula by type of pathology, most of them of primary tract intersphinctertic type (25\%) followed by horseshoe (20\%), and secondary tract intersphincteric (15\%) and transphincteric types (15\%) as shown in Table 1.
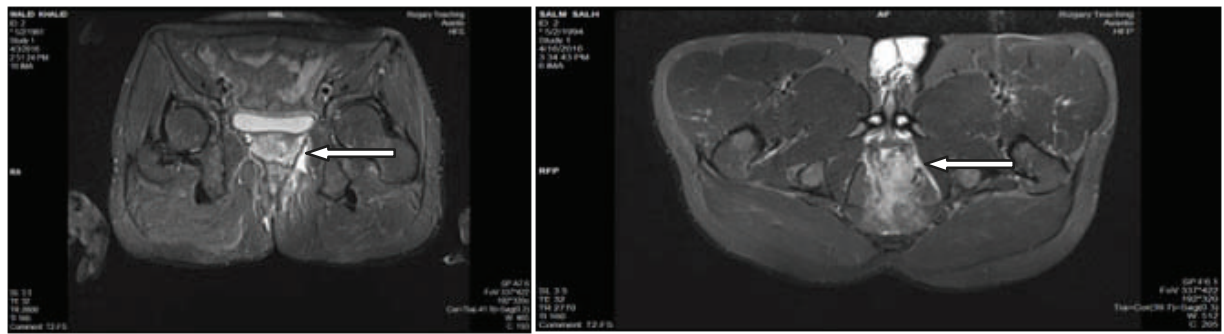

Figure 6: Axial T2 fat suppression weighted images show inflammation and edema in ischiorectal and ischioanal fossa.

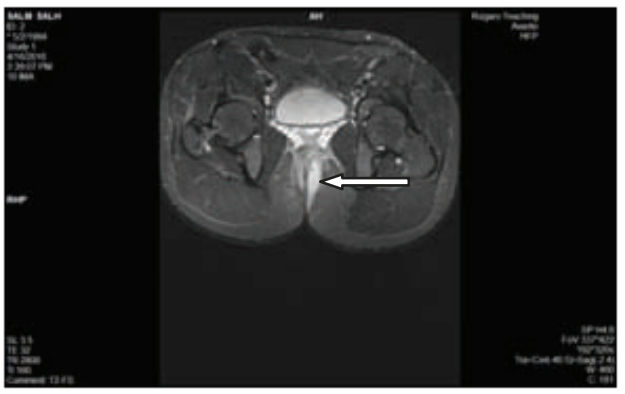

Figure 7: Coronal T2 fat suppression weighted image shows the course of the fistula tract.

Table 1: Distribution of MRI perianal fistula by type of pathology.

\begin{tabular}{lcc}
\hline Type & No. & $\%$ \\
\hline Primary tract & 5 & 25 \\
Intersphincteric & 2 & 10 \\
Tansphincteric & 0 & 0 \\
Supersphincteric & 0 & 0 \\
Extrasphincteric & & \\
Abscess & 1 & 5 \\
Intersphincteric & 1 & 5 \\
Tansphincteric & 4 & $\mathbf{2 0}$ \\
Horse shoe & & 15 \\
Secondary tract & 3 & 15 \\
Intersphincteric & 3 & 0 \\
Tansphincteric & & 10 \\
Granulomatous edema & 0 & \\
Intersphincteric & 2 & \\
Tansphincteric & & \\
\hline
\end{tabular}


Regarding the distribution of perianal fistula by grading, most of them where of Grade 2 (36.8\%) followed by Grade1 (26.3\% and Grade 4 (26.3\%) as shown in Table 2.

\section{Discussion}

Magnetic resonance imaging (MRI) gives a detailed insight into the anatomy of the anal sphincter, especially when high spatial resolution imaging is performed. This study included 20 patients who presented with peri-anal sepsis and visualized external opening, with fistulous tract seen on MRI to detect the course, ramifection and clarify the role of MRI in differentiation between different types of fistulas and detection of associated complications. The age groups included in this study were between 22-55 years with a mean of 38.5 and this agrees with Halligan et al. $^{10}$ who stated that the disease predominantly strikes young adults. However, this finding disagrees with another study that found that perianal fistulae are more common above the age of 35 years old. ${ }^{13}$ Sixteen of 20 patients in our study were male and this agrees with Halligan et al., ${ }^{10}$ who stated that men are predominantly and more commonly affected the disease. Twelve cases had G1 and G2 disease with 60\% were intersphincter, while seven cases had a G3 and G4 disease which represented 35\% transphincter. This finding agrees with another study. ${ }^{11}$ A study done by Charles et al. ${ }^{14}$ showed that T2W images (TSE and fat-suppressed) provide a good contrast between the hyperintense fluid in the tract and the hypointense fibrous wall of the fistula while providing a good delineation of the layers of the anal sphincter. They also found gadolinium-enhanced T1W images are useful to differentiate a fluid-filled tract from an area of inflammation. This agrees with our study as axial T2W fat-suppressed images were the most useful for locating the fistulous tract and after $\mathrm{Gd}$ injection, the tract wall enhances. Abscesses are also very well depicted on post-gadolinium images. Stoker et al. ${ }^{15}$ stated that the internal opening was successfully depicted by FS-CE-T1WI and T2WI and STIR images and this agrees with our study. The course of the tract is more conspicuous in coronal T2 fat suppression and this agrees with results of a study done by Joyce et al. $^{16}$ Complex fistula was more than simple and this agrees with a study from India where Type III and type IV were more common in Indian patients. ${ }^{13}$ This is mostly because patients in the present study underwent MRI examination at a late stage of the disease.

\section{Conclusion}

MRI imaging of perianal fistulae relies on the inherent high soft tissue contrast resolution and the multi-planar display of anatomy by MRI modality. Knowledge of MRI pelvic anatomy is a necessity to recognize the fistulous tracks and their associated ramifications and abscesses. MRI noninvasively offers important information that can reduce surgical complications and postoperative recurrence of the disease. Operating a perianal fistula without an MRI would be

Table 2: Distribution of perianal fistula by grading on $\mathrm{MRI}^{*}$

\begin{tabular}{lcc}
\hline Grade & No. & $\%$ \\
\hline G1 & 5 & 26.3 \\
G2 & 7 & 36.8 \\
G3 & 2 & 10.5 \\
G4 & 5 & 26.3 \\
G5 & 0 & 0 \\
Total & 19 & 100 \\
\hline
\end{tabular}

* One case was perianal sinus, so it is not reported as grade. 
doing a 'blind' and partial management of the disease. This clearly highlights that the important role of MRI has in the management of perianal fistula. MRI has become the most important examination for the evaluation of fistulas and conveying information to clinicians and draws a map especially for surgeons enabling selection of the most appropriate surgical treatment and, therefore, minimizing all chances of recurrence. No other imaging modality can as accurately demonstrate these important pathologies as an MRI examination. Eliminating of the sagittal plane which in turn improves patient co-operation as well improves patient throughput in a busy department. MRI is the gold standard and should be the modality of choice for detail preoperative evaluation of perianal fistulae. Every patient suspected to have perianal fistula should undergo MRI examination to reduce the complications and morbidity.

\section{Conflicts of interest}

The authors report no conflicts of interest.

\section{References}

1. Torkzad MR, Karlbom U. MRI for assessment of anal fistula. Insights Imaging 2010; 1(2):62-71.

2. Joshi AR, Siledar SG. Role of MRI in ano-rectal fistulas. Curr Radiol Rep 2014; 2:63.

3. Abcarian $\mathrm{H}$. Anorectal infection: abscess-fistula. Clin Colon Rectal Surg 2011; 24(1):14-21.

4. Bhaya AK, Kumar N. MRI with MR fi stulogram for perianal fistula: A successful combination. Clin Gastrointest Magnetom 2007; 1:56-9.

5. Poggio JL. Fistula-in-ano. Emedicine; 2015. (Accessed March 12, 2016). Available at: http:// emedicine.medscape.com/article/190234overview).

6. Tabry $\mathrm{H}$, Farrands PA. Update on anal fistulae: surgical perspectives for the gastroenterologist. Can J Gastroenterol 2011; 25(12):675-80.

7. Woodburne RT. Essentials of human anatomy. $7^{\text {th }}$ ed. Oxford, England: Oxford University Press; 1983.

8. Gage KL, Deshmukh S, Macura KJ, Kamel IR, Zaheer A. MRI of perianal fistulas: bridging the radiological-surgical divide. Abdom Imaging 2013; 38(5):1033-42.

9. Alaat El, Essawy MT. Magnetic resonance imaging in assessment of anorectal fistulae and its role in management. J Gastroint Dig Syst 2013; 3:139.

10. Halligan S, Stoker J. Imaging of fistula in ano. Radiology 2006; 239:18-33.
11. Morris J, Spencer JA, Ambrose NS. MR imaging classification of perianal fistulas and its implications for patient management. Radiographics 2000; 20:623-35.

12. Park AG, Gordon PH, Hardcastle JD. A classification of fistula-in-ano. Br J Surg 1976; 63:1-12.

13. Adurthy $\mathrm{P}$, Prabhu SD, Kumar A. MRI in Assessment of Perianal Fistula. Indian J Applied Research 2016; 6(1):502-3.

14. de Miguel Criado J, del Salto LG, Rivas PF, del Hoyo LF, Velasco LG, de las Vacas MI, et al. MR Imaging Evaluation of Perianal Fistulas: Spectrum of Imaging Features. Radiographics 2012; 32(1):175-94.

15. Stoker J, Rociu E, Wiersma T, Lame' ris J. Imaging of anorectal disease. $\mathrm{Br} \mathrm{J}$ Surg 2000; 87:10-27.

16. Joyce $M$, Veniero $J$, Pokala R. Magnetic resonance imaging in the management of anal fistula and anorectal sepsis. Clin Colon Rectal Surg 2008; 21(3):213-9. 